\title{
XVe Table Ronde sur les problèmes actuels du droit international humanitaire
}

(San Remo, Italie, 4-8 septembre 1990)

La traditionnelle Table Ronde de San Remo sera organisée du 4 au 8 septembre 1990 par l'Institut international de droit humanitaire (IIDH)* et sera placée sous le patronage du Comité international de la Croix-Rouge, du Haut Commissariat des Nations Unies pour les réfugiés, du Centre des Droits de l'Homme des Nations Unies, de l'Organisation internationale pour les Migrations et de la Ligue des Sociétés de la Croix-Rouge et du Croissant-Rouge.

Les hauts représentants de ces organisations internationales ainsi que des experts gouvernementaux et des spécialistes provenant de toutes les régions du monde participeront à ce forum humanitaire qui, dans le cadre d'un dialogue ouvert et informel, permet traditionnellement de développer la réflexion sur les problèmes humanitaires actuels.

Les principaux thèmes de la Table Ronde seront les suivants: Obligation des Etats tiers de faire respecter le droit international humanitaire (DIH) (Art. 1 commun aux quatre Conventions de Genève), rôle des Nations Unies, procédures d'enquête, compétence de la Commission internationale d'établissement des faits (Art. 90, Protocole I) et utilité d'un système de rapports périodiques sur l'application du DIH.

Le Symposium de la Croix-Rouge et du Croissant-Rouge sera consacré à l'incidence des législations et politiques nationales sur le développement des Sociétés nationales de la Croix-Rouge et du Croissant-Rouge.

La Journée des réfugiés traitera du thème suivant: «Réfugiés - un défi permanent pour l'humanité: pour une approche réaliste de la politique d'asile". (Italie).

* Secrétariat de l'IIDH: Villa Ormond, Corso Cavallotti 112, 18038 San Remo 
Enfin, l'Institut international de droit humanitaire célèbrera son vingtième anniversaire par un concert de l'Orchestre symphonique de San Remo, avec la participation de Mme Barbara Hendricks, soprano et de M. Justus Frantz, pianiste, tous deux Ambassadeurs de Bonne Volonté du HCR.

Un compte rendu des travaux de la Table Ronde paraîtra dans un des prochains numéros de la Revue.

Dans le prochain numéro (septembre-octobre) 1990 de la Revue internationale de la Croix-Rouge:

- Règles du droit international humanitaire relatives à la conduite des hostilités dans les conflits armés non internationaux

- La répression pénale des violations du droit international humanitaire applicable aux conflits armés non internationaux

- Enfants-combattants prisonniers 